Talk presented at the 6th International Conference on Large Scale Applications and Radiation Hardness of Semiconductor Detectors, September 29-October 12003 , Firenze, Italy.

\title{
Tests of silicon sensors for the CMS pixel detector
}

\author{
A. Dorokhov a,b,1, C. Amsler ${ }^{a}$, D. Bortoletto ${ }^{\text {c }}$, V. Chiochia ${ }^{\text {a }}$, \\ L. Cremaldi ${ }^{\text {d }}$, S. Cucciarelli ${ }^{\text {e }}$, M. Konecki ${ }^{\mathrm{e}}, \mathrm{K}$. Prokofiev ${ }^{\mathrm{a}, \mathrm{b}}$, \\ C. Regenfus ${ }^{\text {a }}$, T. Rohe ${ }^{\text {b }}$, D. Sanders ${ }^{d}$, S. Son ${ }^{\text {c }}$, T. Speer ${ }^{a}$, \\ M. Swartz ${ }^{\mathrm{f}}$ \\ a Physik Institut der Universität Zürich-Irchel, 8057 Zürich, Switzerland \\ ${ }^{\mathrm{b}}$ Paul Scherrer Institut, 5232 Villingen PSI, Switzerland \\ ${ }^{\mathrm{c}}$ Purdue University, Task G, West Lafayette, IN 47907, USA \\ d Mississippi State Univ., Department of Physics and Astronomy, MS 39762, USA \\ ${ }^{\mathrm{e}}$ Institut für Physik der Universität Basel, Basel, Switzerland \\ ${ }^{\mathrm{f}}$ Johns Hopkins University, Baltimore, MD, USA
}

\begin{abstract}
The tracking system of the CMS experiment, currently under construction at the Large Hadron Collider (LHC) at CERN (Geneva, Switzerland), will include a silicon pixel detector providing three spacial measurements in its final configuration for tracks produced in high energy $p p$ collisions. In this paper we present the results of test beam measurements performed at CERN on irradiated silicon pixel sensors. Lorentz angle and charge collection efficiency were measured for two sensor designs and at various bias voltages.
\end{abstract}

Key words: Pixel; Radiation hardness; Lorentz angle; Charge collection; CMS; LHC

\section{Introduction}

The CMS experiment at the Large Hadron Collider (LHC) at CERN (Geneva, Switzerland) will be equipped with a silicon pixel detector providing high resolution 3D coor-

$\overline{1}$ Corresponding author. E-mail address: Andrei.Dorokhov@cern.ch (A.Dorokhov).

Preprint submitted to Elsevier Preprint dinates of the tracks. It will consist of three layers of pixel sensors $(100 \mu \mathrm{m} \times 150 \mu \mathrm{m}$ pitch size $)$ in the barrel region and two discs in the forward regions [1]. The innermost barrel layer is expected to be exposed to a fluence ${ }^{2}$ of $3 \times 10^{14} \mathrm{n}_{\mathrm{eq}} / \mathrm{cm}^{2}$ per year at full luminosity. The

2 All particle fluences are normalized to $1 \mathrm{MeV}$ neutrons $\left(\mathrm{n}_{\mathrm{eq}} / \mathrm{cm}^{2}\right)$. 
second and third layer will be exposed to $1.4 \times 10^{14} \mathrm{n}_{\mathrm{eq}} / \mathrm{cm}^{2}$ and $0.6 \times 10^{14} \mathrm{n}_{\mathrm{eq}} / \mathrm{cm}^{2}$ per year, respectively. The charge sharing induced by the Lorentz force in the $4 \mathrm{~T}$ CMS detector magnetic field can improve the spatial resolution. Hence it is very important to study the effects of charge collection efficiency and Lorentz drift in irradiated silicon devices. Here we present measurements of Lorentz angle and charge collection efficiency, and compare two sensor designs at different irradiation doses.

\section{Experimental setup}

The measurements were performed in the $\mathrm{H} 2$ beam line of the CERN SPS in June and September 2003 using $150-225 \mathrm{GeV}$ pions. A silicon reference telescope [2] was used to allow a precise determination of the particle hit position in the pixel detector. Both, the telescope modules and the pixel front-end, were mounted onto a common frame (see fig. 1). The beam telescope consists of 4 modules. Each consists of two $300 \mu \mathrm{m}$ thick singlesided silicon detectors $\left(32 \times 30 \mathrm{~mm}^{2}\right)$ with a strip pitch of $25 \mu \mathrm{m}$ (readout pitch of $50 \mu \mathrm{m}$ ), which are oriented perpendicularly. The resulting intrinsic resolution of the beam telescope is around $1 \mu \mathrm{m}$.

The pixel sensor with the readout chip is mounted on a rotating support positioned between the second and the third module. A trigger signal is generated by a silicon PIN diode. The data acquisition system, including
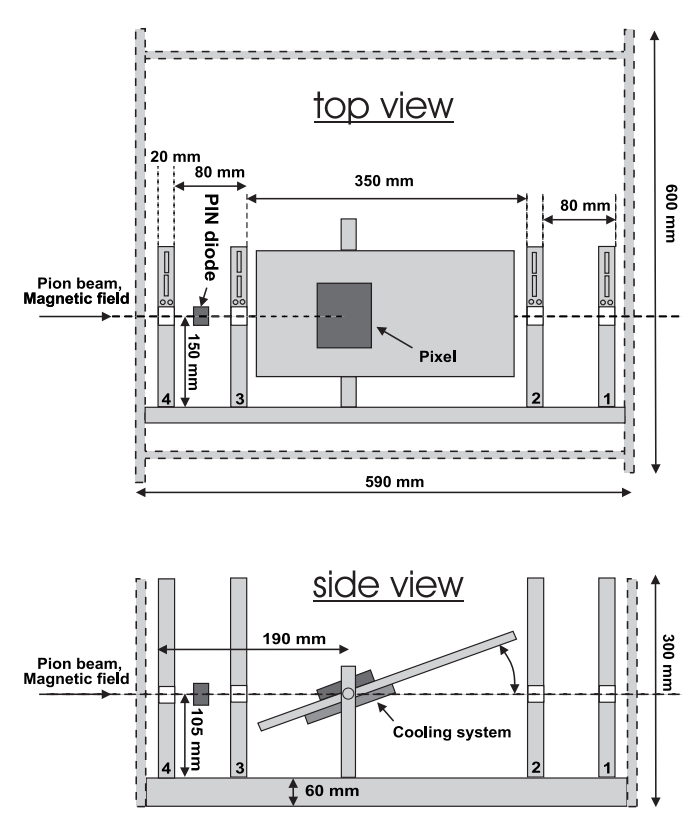

Fig. 1. Top and side views of the beam telescope.

slow control (temperature and bias voltage of the pixel sensor) is written in LabView and LabWindows CVI (National Instruments) running on a PC. The analog signals are digitized in a VME based readout system by two CAEN (V550) and one custom built FADCs [3]. The whole setup was placed in a open $3 \mathrm{~T}$ Helmholtz magnet with magnetic field parallel to the beam. The pixel sensors were cooled down up to $-30^{\circ} \mathrm{C}$ by means of water cooled Peltier elements.

\section{Pixel sensors and front-end electronics}

The CMS pixel sensors are manufactured in the "n-on-n" technique, consisting of $\mathrm{n}^{+}$structures on $\mathrm{n}$ bulk silicon. This allows a partially depleted operation of highly irradiated sensors after type inversion, but re- 
quires inter-pixel isolation. Two isolation techniques were considered in our latest prototype designs: pspray [4], where a uniform medium dose of p-impurities covers the whole structured surface, and p-stop, where higher dose rings individually surround the $\mathrm{n}^{+}$-implants. Fig. 2 shows the layouts of these designs. Due to possible failures in the pixel contacts (bump-bonding) an intrinsic biasing method has to be introduced. This is realized by a bias grid and punchtrough structures [5] for the p-spray detectors and by openings in the rings for the p-stop detectors. All our

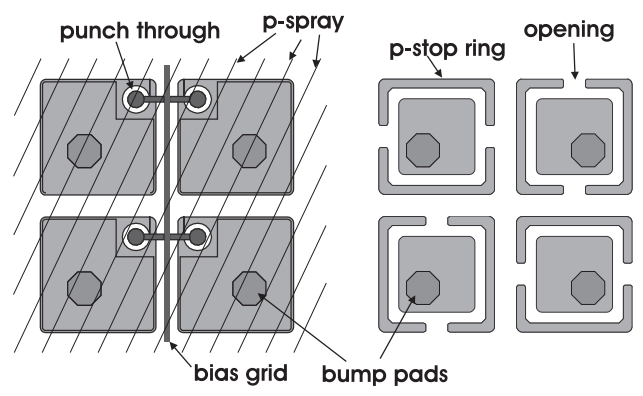

Fig. 2. Layout of the pixel sensors under study: p-spray with bias grid (left) and open p-stop rings (right).

test devices had $22 \times 32$ pixels, with a sensitive area of $2.75 \times 4 \mathrm{~mm}^{2}$, and a thickness of $280 \mu \mathrm{m}$. The readout pitch was $125 \mu \mathrm{m} \times 125 \mu \mathrm{m}$.

Some of the devices were irradiated in a $24 \mathrm{GeV}$ proton beam at the CERN PS. The sensors received total particle fluences of $3.3 \times 10^{14} \mathrm{n}_{\text {eq }} / \mathrm{cm}^{2}, 8.1 \times 10^{14} \mathrm{n}_{\text {eq }} / \mathrm{cm}^{2}$ and $1.1 \times 10^{15} \mathrm{n}_{\mathrm{eq}} / \mathrm{cm}^{2}$ respectively. During the irradiation the sensors were kept at room temperature and without bias, while after irradiation we stored and operated them at $-20^{\circ} \mathrm{C} . I V$-curves were measured [6] before and after irradiation, all mea- sured sensors showed a breakdown voltage above $600 \mathrm{~V}$. Finally the sensors were bump bonded to non irradiated readout chips of the type PSI30/AC30 [7] allowing for a non zero-suppressed analog readout of all 704 pixel cells. A fast (20 ns) external signal for the internal sample and hold mechanism had to be provided from the trigger PIN diode.

\section{Beam test measurements}

\subsection{Lorentz angle measurements}

The Lorentz angle is obtained by the direct measurement of the charge drift in the magnetic field using the grazing angle method [8]. The par-

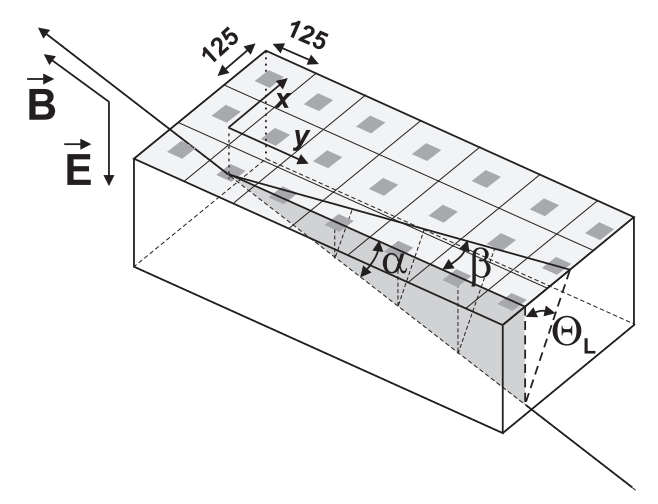

Fig. 3. Lorentz angle measurement with the grazing angle method.

ticle beam (parallel to the magnetic field) hits the pixel surface at a shallow angle $\alpha=15^{\circ}$ (see fig. 3). The deposited charge drifts according to the combined electric and magnetic forces, resulting in a deflection of the particle track projection on the surface by the angle $\beta$. The Lorentz angle is obtained from the relation 
$\tan \Theta_{L}=\tan \beta / \tan \alpha$.

The deflection measured at $3 \mathrm{~T}$ magnetic field is shown in fig. 4 for the non-irradiated p-spray sensor. The

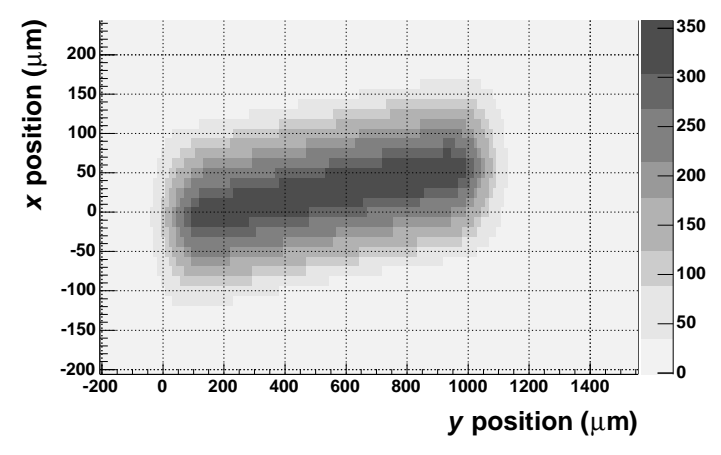

Fig. 4. Deflection of the collected charge in a $3 \mathrm{~T}$ magnetic field.

angle $\beta$ is measured by slicing the histogram perpendicularly to the $y$ axis. The position of the center of each slice is shown in fig. 5 as a function of the position along the $y$-axis. A measurement without magnetic field is used to correct for the detector misalignment with respect to the beam (bottom line in fig. 5). For the irradiated device in fig. 5 there are two regions with a different slope, and hence, two different values of the Lorentz angle. The difference in the Lorentz angle can be explained by nonuniform distribution of the electric field in the irradiated devices. Different models of the electric field distribution in the irradiated silicon devices are discussed in Refs. $[9,10]$. The evidence of the non-uniform electric filed will be shown also in the next section.

As most of the signal charge is collected from the region close to the pixel implant, this region was used to determine the Lorentz angle. Figure 6 shows the measured values for

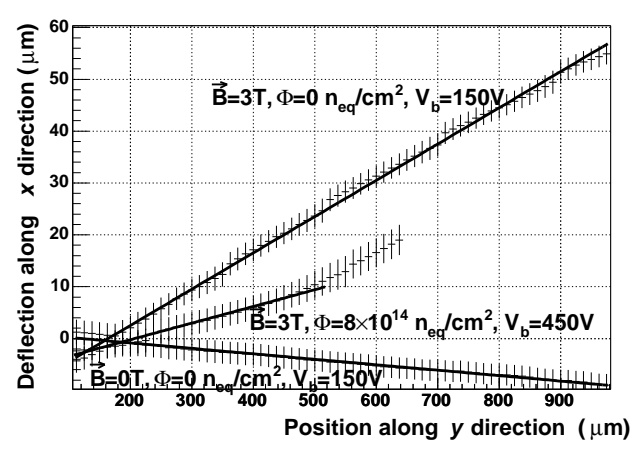

Fig. 5. Deflection of the collected charge as a function of the $y$-position(solid line is fit).

both non-irradiated and irradiated sensors extrapolated to $4 \mathrm{~T}$ magnetic field. We observe a strong depen-

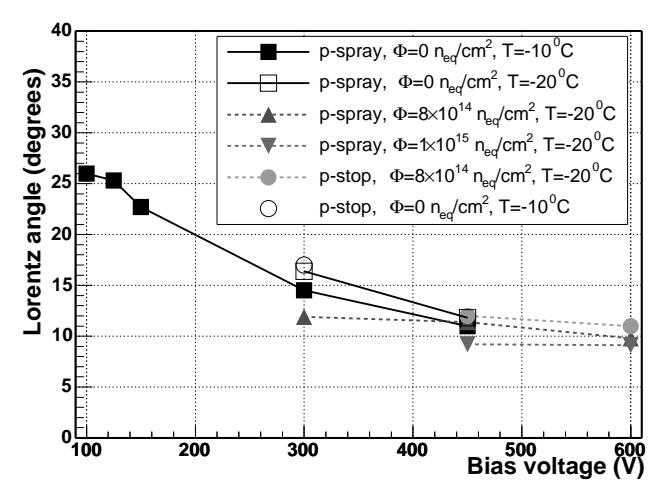

Fig. 6. Lorentz angle as a function of the bias voltage for $4 \mathrm{~T}$ magnetic field.

dence of the Lorentz angle on the bias voltage (electric field), while it is only weakly affected by the irradiation or sensor design. For the nonirradiated sensors a Lorentz angle of $26^{\circ}$ can be reached at a bias voltage of $100 \mathrm{~V}$, while irradiated sensors have to be operated at higher bias voltages, where the Lorentz angle drops to roughly $10^{\circ}$. Since the electron mobility increases with decreasing temperature the Lorentz angle measured at the lower temperature $-20^{\circ} \mathrm{C}$ is $1^{\circ}-2^{\circ}$ larger. Our values 
for the Lorentz angle are in a good agreement with measurements and simulations in Refs. [11,12].

\subsection{Charge collection in irradiated sensors}

After irradiation the collected charge decreases due to charge trapping and partial depletion of the sensor. The measurements of the charge collection efficiency as a function of the sensor depth were performed using the grazing angle method without magnetic field. The averaged charge cluster profiles in non-irradiated and irradiated p-spray sensors are shown in fig. 7. In the unirradiated sensor the charge is collected uniformly across the whole sensor depth, while the irradiated devices shows two regions with different collected charge. At low bias voltage in the irradiated detectors some charge is also collected from the side opposite to the pixel implants. This can indicate that the depletion starts from both sides of the detector, because of the nonuniform distribution of the electric filed. The integral of the collected

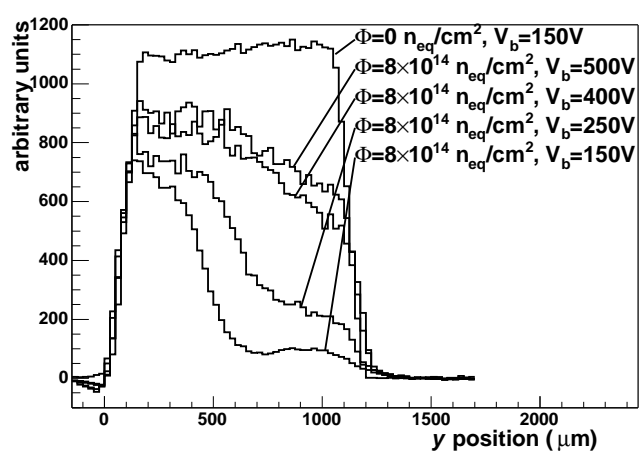

Fig. 7. Charge cluster for different bias voltages. charge from the particle entry point up to a point along the $y$-coordinate is shown in fig. 8. There are two regions with different charge collection, represented by two different slopes of the curve for the irradiated device. The total collected charge for differ-

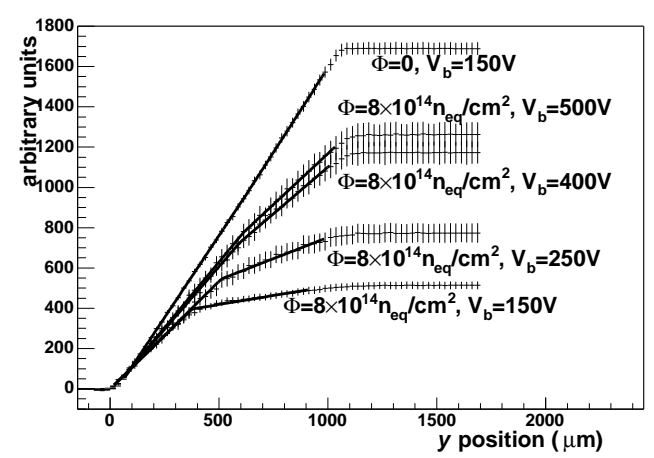

Fig. 8. Integrated charge as a function of the distance to the particle entry point.

ent designs and irradiation doses as a function of bias voltage is shown in fig. 9 .

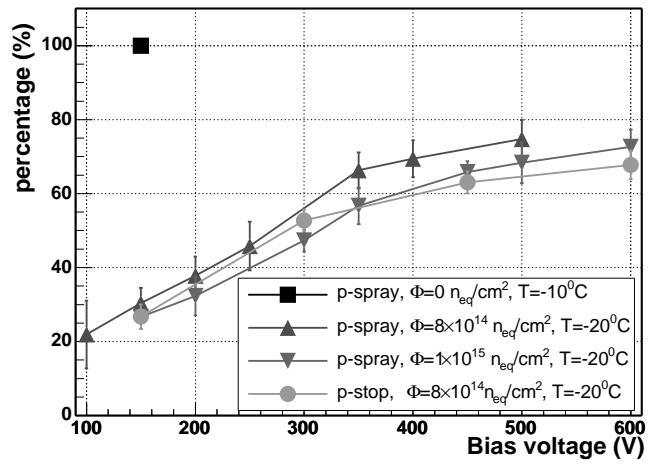

Fig. 9. Total collected charge normalized to non-irradiated device.

\subsection{Signal-to-noise ratio}

The charge collection depends on the position of the incident particle with respect to the pixel implant, caused 

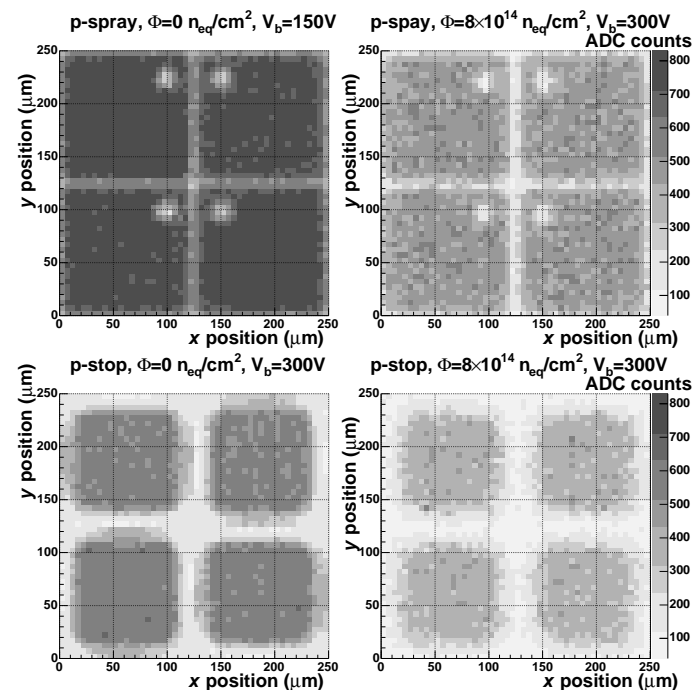

Fig. 10. Charge collected in the hit pixel for non irradiated (left) and irradiated sensors (right).

by the regions with reduced sensitivity. The average charge collected in the hit pixel as a function of the hit position is shown in fig. 10 for nonirradiated and irradiated sensors. One can see that after irradiation the average pixel signal decreases, and the area with the reduced charge collection increases in the case of the p-stop design.

The signal-to-noise ratio $(\mathrm{S} / \mathrm{N})$ averaged over the whole pixel area is shown in fig. 11. The measured signal-to-noise ratio is above 30 even after the irradiation. We observe lower $\mathrm{S} / \mathrm{N}$ ratio in the unirradiated p-stop device, because of the charge spread over several pixels caused by the p-stop openings. A more detailed study of charge collection for these sensors can be found in Ref. [6].

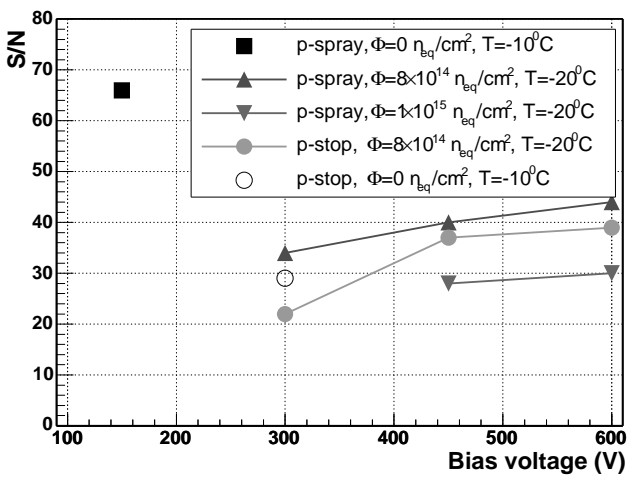

Fig. 11. Signal-to-noise ratio as a function of the bias voltage.

\section{Conclusions}

Two different designs of the CMS prototype pixels sensors were tested up to $600 \mathrm{~V}$ bias voltage and after exposure to particle fluences up to $1.1 \times 10^{15} \mathrm{n}_{\mathrm{eq}} / \mathrm{cm}^{2}$. For the irradiated devices we observe two regions across the sensor with different charge collection and value of the Lorentz angle. This result is an indication of a nonuniform electric field in the irradiated devices.

The Lorentz angle mainly depends on the bias voltage and it shows a weak dependence on the irradiation dose or sensor design. The Lorentz angle at $4 \mathrm{~T}$ magnetic field reaches $26^{\circ}$ for the non-irradiated devices at a bias voltage of $100 \mathrm{~V}$ and it drops to $8.3^{\circ}$ for the ones irradiated at $1.1 \times 10^{15} \mathrm{n}_{\mathrm{eq}} / \mathrm{cm}^{2}$ and at a bias voltage $600 \mathrm{~V}$.

After irradiation and at high bias voltage $(600 \mathrm{~V})$ the charge collection is about $60 \%$ of the value observed for the non-irradiated devices, and it is slightly larger for the p-spray design.

The signal-to-noise ratio decreases 
from 65 to about 35 after irradiation giving us confidence in operating the CMS pixel detector up to the maximum expected irradiation dose.

For the irradiated devices the charge collection, and therefore the signal height, increases with the bias voltage but the Lorentz angle decreases. For this reason the bias voltage must be optimized for the best performance of the CMS pixel detector.

\section{Acknowledgments}

We gratefully acknowledge Silvan Streuli from ETH Zurich and Fredy Glaus from PSI for their immense effort on the bump bonding, Maurice Glaser and Michael Moll from CERN for carrying out the irradiation, Kurt Bösiger from the Zürich workshop for the mechanical construction, György Bencze and Pascal Petiot from CERN for the $\mathrm{H} 2$ beam line support and finally the whole CERN-SPS team. The authors would like to thank Dr. Danek Kotlinski from PSI for very useful discussions.

\section{References}

[1] CMS Collaboration, CMS TDR 5, CERN-LHCC-1998-06, 1998.

[2] C. Amsler et al., Nucl. Instr. and Meth. in Phys. Research A 480 (2002) 501.

[3] M. Pernicka, wwwhephy.oeaw.ac.at/p3w/
electronic2/ELEK2/ARCHIV/CMS/

PIX_READ_SYS/pxrd_sys_js.html

[4] R. H. Richter et al., Nucl. Instr. and Meth. in Phys. Research A $\mathbf{3 7 7}$ (1996) 412.

[5] T. Rohe et al., Nucl. Instr. and Meth. in Phys. Research A 409 (1998) 224.

[6] T. Rohe et al., IEEE-NSS, October 19-25, 2003, Portland, Oregon, USA, to be published.

[7] D. Meer, Bau und Messen eines Multichip Pixelmodules als Prototyp für den CMS Traker, Diploma Thesis, ETH Zürich, March 2000.

[8] B. Henrich, R. Kaufmann, Nucl. Instr. and Meth. in Phys. Research A 477 (2002) 304.

[9] V. Eremin, E. Verbitskaya, Z. Li Nucl. Instr. and Meth. in Phys. Research A 476 (2002) 556.

[10] A. Castaldini et al., Journal of Applied Physics Vol 92(4) pp. 20132016. August 15, 2002.

[11] V. Bartsch et al., Nucl. Instr. and Meth. in Phys. Research A $\mathbf{4 7 8}$ (2002) 330.

[12] V. Bartsch et al., Nucl. Instr. and Meth. in Phys. Research A 497 (2003) 389. 